\title{
PACE4 regulates proliferation, migration and invasion in human breast cancer MDA-MB-231 cells
}

\author{
FEIFEI WANG ${ }^{1-3}$, LIN WANG ${ }^{1,2}$ and JIHONG PAN ${ }^{1,2}$ \\ ${ }^{1}$ Shandong Medicinal and Biotechnology Center, Shandong Academy of Medical Sciences; ${ }^{2}$ Key Laboratory for Biotech-Drugs, \\ Ministry of Health, Key Laboratory for Rare and Uncommon Diseases of Shandong Province; \\ ${ }^{3}$ College of Medical and Life Sciences, Jinan University and Shandong Academy of Medical Sciences, \\ Jinan, Shandong 250062, P.R. China
}

Received November 7, 2013; Accepted July 21, 2014

DOI: $10.3892 / \mathrm{mmr} .2014 .2691$

\begin{abstract}
PACE4 is one of the proprotein convertases (PC) that participate in the post-translational activation of inactive proteins, leading to mature, biologically active proteins. The processing reactions occur in pairs of basic amino acids. PACE4 is an extracellular PC that binds to growth factors and several components of the extracellular matrix contributing to tumor progression. In the present study, the PACE4 gene was silenced by small interfering RNA (siRNA), and the knockdown human breast cancer MDA-MB-231 cells showed significantly reduced proliferation, migration and invasion rates. Flow cytometry analysis indicated that downregulation of PACE4 increases the percentage of cells arrested at the G0/G1 phase. Moreover, the expression of genes involved in cell growth, invasion and adhesion, i.e., IGF-2, MMP9 and MPZL2 was significantly decreased following siRNA-mediated silencing of PACE4. Taken together, these results indicate that PACE4 plays an important role in human breast cancer, and that it might represent a novel target for breast cancer therapy.
\end{abstract}

\section{Introduction}

PACE4 is one of the neuroendocrine-specific mammalian subtilisin-related endoproteases and a calcium-dependent serine proteinase. PACE4 can cleave precursor proteins at basic residues within the motif $(K / R)-(X) n-(K / R) \downarrow$, where $n=0$, 2, 4, 6 and $X$ is any amino acid except Cys (1). Eight isoforms of PACE4 have been reported: PACE4A-I, PACE4A-II, PACE4B, PACE4C, PACE4CS, PACE4D, PACE4 E-I and PACE4E-II (2). These isoforms are distributed in different

Correspondence to: Dr Jihong Pan, Shandong Medicinal and Biotechnology Center, Shandong Academy of Medical Sciences, 18877 Jingshi Road, Jinan, Shandong 250062, P.R. China

E-mail: pjh933@sohu.com

Key words: human breast cancer cells, PACE4, proliferation, migration, invasion, cell cycle tissues in humans, and have been shown to function in the secretory pathway (3).

Breast cancer has become a common tumor, threatening women's physical and mental health. It is a complex tumor, and one of its most common causes is genetic mutations. A number of reports have provided evidence for an association betweengenes such as BRCA-1, BRCA-2, p53, PTEN etc., with breast cancer $(4,5)$. The common features of cancer cells, namely, increased proliferation, migration and tissue invasion, lead to uncontrolled division and allow cells to invade surrounding tissues as well as basement membranes, thereby accelerating malignant growth and cancer progression. The protein PACE4 is strongly involved in malignancy, due to its activity that allows to generate biologically active proteins. There is evidence that aberrant expression of PACE4 is involved in different types of cancer. For example, overexpression of PACE4 in murine cells leads to increased invasiveness both in vitro and in vivo (6), and enhances tumor malignant phenotypes in breast cancer (7). Another report indicated that PACE4 plays an important role in enhancing the progression of prostate cancer (8). The critical role of PACE4 in promoting proceedings of tumors renders it an attractive target for cancer treatment.

PACE4 was shown to play a pivotal role in the extracellular matrix (ECM) (9). Numerous proteins such as growth factors, cell adhesion molecules, and oncogene products are active in the ECM. All these proteins are important in the occurrence and progression of cancer. Thus, PACE4 may also be involved in cancer. A previous study indicated that $\alpha 1-\mathrm{PDX}$ and ppPACE4 (PACE4 prosegment) can increase cell motility, migration and invasion (7). In this context, we aimed to explore the direct effect of PACE4 in breast cancer cells and further investigate the roles of PACE4 in this type of cancer.

In the present study, an RNA interference approach was adopted to silence the expression of the PACE4 gene in the MDA-MB-231 cell line and thereby, explore the role of PACE4 in breast cancer. We studied the proliferation, migration and invasion of MDA-MB-231 cells following siRNA transfection, and also detected cell-cycle changes following the reduction in the expression of PACE4. Breast cancer is a tumor related to hormones, and thus, the relationship between PACE4 and $17 \beta$-estradiol was also investigated. In order to elucidate the 
mechanism underlying the PACE4 effects on MDA-MB-231 cells, the expression of genes involved in cell growth, invasion and adhesion, such as matrix metalloproteinase-9 (MMP-9), insulin like growth factor $2(I G F-2)$ and myelin protein zero-like 2 (MPZL2), was also studied following siRNAmediated inhibition of PACE4 expression.

\section{Materials and methods}

Cell culture. Human breast cancer MDA-MB-231 and MCF-7 cells were obtained from the Shanghai Institutes for Biological Sciences (Shanghai, China) and cultured in HyClone ${ }^{\mathrm{TM}}$ Dulbecco's modified Eagle's medium (DMEM) containing $10 \%$ HyClone $^{\mathrm{TM}}$ fetal bovine serum (HyClone), as well as Gibco ${ }^{\circledR}$ penicillin $(100 \mathrm{IU} / \mathrm{ml})$ and streptomycin $(100 \mu \mathrm{g} / \mathrm{ml})$ in a $37^{\circ} \mathrm{C}, 5 \% \mathrm{CO}_{2}$ incubator (all from Thermo Fisher Scientific, Rockford, IL, USA).

Small interfering RNA transfection. Three pairs of siRNAs were used in order to achieve the most effective inhibition of PACE4 expression (si-PACE4-1 to -3). The siRNAs were synthesized by GenePharma (Shanghai, China) and target the human PACE4 transcript (GenBank accession no., NM_002570.3), at positions 1,011, 1,196, and 2,118 of the sequence. The cultured MDA-MB-231 cells were transfected with the siRNAs using the HiPerFect transfection reagent (Qiagen, Hilden, Germany) according to the manufacturer's protocol, and three siRNA concentrations $(50,100$ and $150 \mathrm{nM}$ ) were tested. Cells transfected with an unrelated siRNA pair (5'-CUCCGAACGUGUCACGUTT-3') and cells cultured with the transfection reagent alone were used as the negative control (NC) and the mock, respectively.

RNA extraction and reverse transcription-quantitative $P C R(R T-q P C R)$. Total RNA was isolated from the cells using the E.Z.N.A. ${ }^{\circledR}$ Total RNA Kit II (Omega Bio-Tek, Norcross, GA, USA) according to the manufacturer's protocol. The extracted RNA was reverse transcribed using the ReverTra Ace ${ }^{\circledR}$ qPCR RT kit (Toyobo, Osaka, Japan) according to the manufacturer's protocol. The relative expression of PACE4 following siRNA transfection was analyzed by qPCR. The PCR was performed three times using the SYBR Green kit (Roche Diagnostics, Mannheim, Germany) and the following primers: PACE4 forward (F), 5'-AAG CAA GGG AAG TTG AAA GA-3', and reverse (R), 5'-CAC TGA AGG TGT GGT ACG-3'; glyceraldehyde 3-phosphate dehydrogenase (GAPDH; used to standardize the level of expression of PACE4) F, 5'-CAC CAT CTT CCA GGA GC-3', and R, 5'-AGT GGA CTC CAC GAC-3'; MMP9 F, 5'-CCC TGC CAG TTT CCA TT-3', and R, 5'-CCA TCA CCG TCG AGT CA-3'; IGF-2 F, 5'-GCT TCT ACT TCA GCA GGC-3', and R, 5'-GTC CCT CTC GGA CTT GG-3'; MPZL2 F, 5'-CTC TAA CAG TGA CCT GGA ATT T-3', and R, 5'-GAA GGA TGG AGG CAT CG-3'. The qPCR reactions were performed on a LightCycler 480 (Roche Diagnostics GmbH, Mannheim, Germany), with the following cycling conditions: an initial denaturation step at $95^{\circ} \mathrm{C}$ for $15 \mathrm{sec}, 45$ cycles at $95^{\circ} \mathrm{C}$ for $10 \mathrm{sec}$ and $60^{\circ} \mathrm{C}$ for $30 \mathrm{sec}$, followed by $10 \mathrm{sec}$ at $72^{\circ} \mathrm{C}$ for final extension. The comparative threshold cycle $(\mathrm{Ct})$ method was used to analyze the relative expression of mRNA. PCR products were subjected to $1 \%$ agarose gel electrophoresis to confirm the specificity of the amplification.

Western blotting. Total protein was extracted by incubation on ice for 30 min with RIPA lysis buffer (Beyotime Institute of Biotechnology, Jiangsu, China) containing protease inhibitors. The preparation was centrifuged at $14,000 \mathrm{x}$ g for $30 \mathrm{~min}$ at $4^{\circ} \mathrm{C}$. The protein concentration was measured with the Bio-Rad protein assay system (Hercules, CA, USA). Following incubation at $100^{\circ} \mathrm{C}$ for $5 \mathrm{~min}$ with loading buffer, $30 \mu \mathrm{g}$ of the total protein were resolved by sodium dodecyl sulfate-polyacrylamide gel electrophoresis, transferred to nitrocellulose membranes (Amersham, Piscataway, NJ, USA) by wet transfer, and blocked in $10 \mathrm{nM}$ Tris-buffered saline (TBS) containing $0.1 \%(\mathrm{v} / \mathrm{v})$ Tween-20 (TBST) and 5\% (w/v) skim milk. Next, the membranes were incubated overnight at $4^{\circ} \mathrm{C}$ with specific anti-human primary antibodies (anti-PACE4 at 2,000-fold dilution, or anti-GAPDH at 1,000-fold dilution). Following a wash with phosphate-buffered saline (PBS) containing $0.1 \%(\mathrm{v} / \mathrm{v})$ Tween-20 (PBST), the membranes were incubated with the corresponding secondary antibody (goat anti-rabbit immunoglobulin G-HRP; 1:5,000 dilution) for $1 \mathrm{~h}$ at $37^{\circ} \mathrm{C}$. All antibodies were purchased from Abcam (Cambridge, UK). Detection was performed using an enhanced chemiluminescence (ECL) kit (KeyGen Biotech, Nanjing, China). The film was scanned and analyzed with Quantity One Analysis software (Bio-Rad).

Cell proliferation assay. The human breast cancer MDA-MB-231 cells were seeded onto 96-well culture plates and incubated until they reached $80 \%$ confluence. The cultured cells were transfected with si-PACE4 $(150 \mathrm{nM})$. After growing for 24, 48 and $72 \mathrm{~h}, 20 \mu \mathrm{l}$ of 3-(4,5-dimethylthiazol-2-yl)2,5-diphenyltetrazolium bromide (MTT; $5 \mathrm{mg} / \mathrm{ml}$ ) were added to each well, and the incubation was continued for an additional $4 \mathrm{~h}$ at $37^{\circ} \mathrm{C}$. Then, the MTT solution was removed, and $150 \mu \mathrm{l}$ of dimethyl sulfoxide (Solarbio, Beijing, China) were added and incubated at room temperature for $10 \mathrm{~min}$ to allow extraction of the MTT-formazan products. The absorbance was measured at $490 \mathrm{~nm}$ with a UV/VIS spectrophotometer (Yuanxi-UV5500, Shanghai, China) and the measurements were repeated three times.

Cell migration and invasion assays. Cell migration was measured by a wound healing assay. The cells were plated onto 24-well plates and scratched linearly in multiple areas with a cell scraper. Then, the cells were transfected with $150 \mathrm{nM}$ siRNA targeting PACE4, or with the negative and the transfection reagent control. The same area of each wound was examined at 0 and $24 \mathrm{~h}$ to quantify the cells that had migrated into the wound.

The cell invasion assay was performed in a Costar ${ }^{\circledR}$ Transwell appparatus coated with a Matrigel filter (Corning, NY, USA). Cells were plated at a density of $3 \times 10^{4}$ cells/well, and incubated with $150 \mathrm{nM}$ of siRNA in the upper chamber of the apparatus for $8 \mathrm{~h}$, while the lower compartment was filled with DMEM with $10 \%$ FBS. The upper and the lower chamber were filled with DMEM without FBS and incubated for $12 \mathrm{~h}$. The non-invading cells at the upper surface of the filter were scraped with cotton swabs, and the invading cells were stained Giemsa 
A

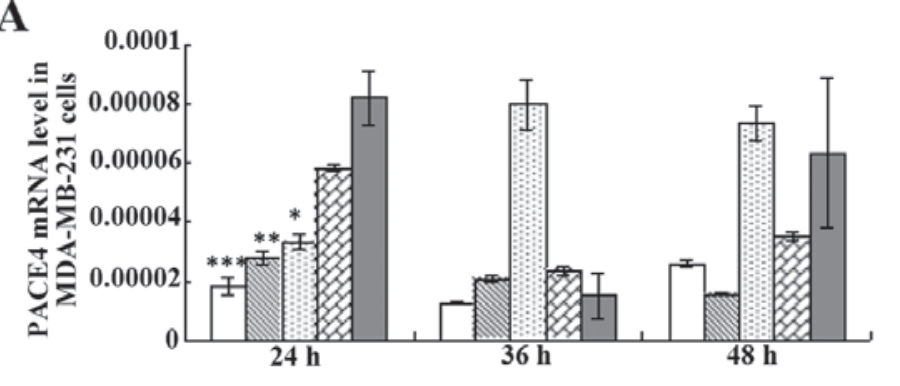

\section{B}
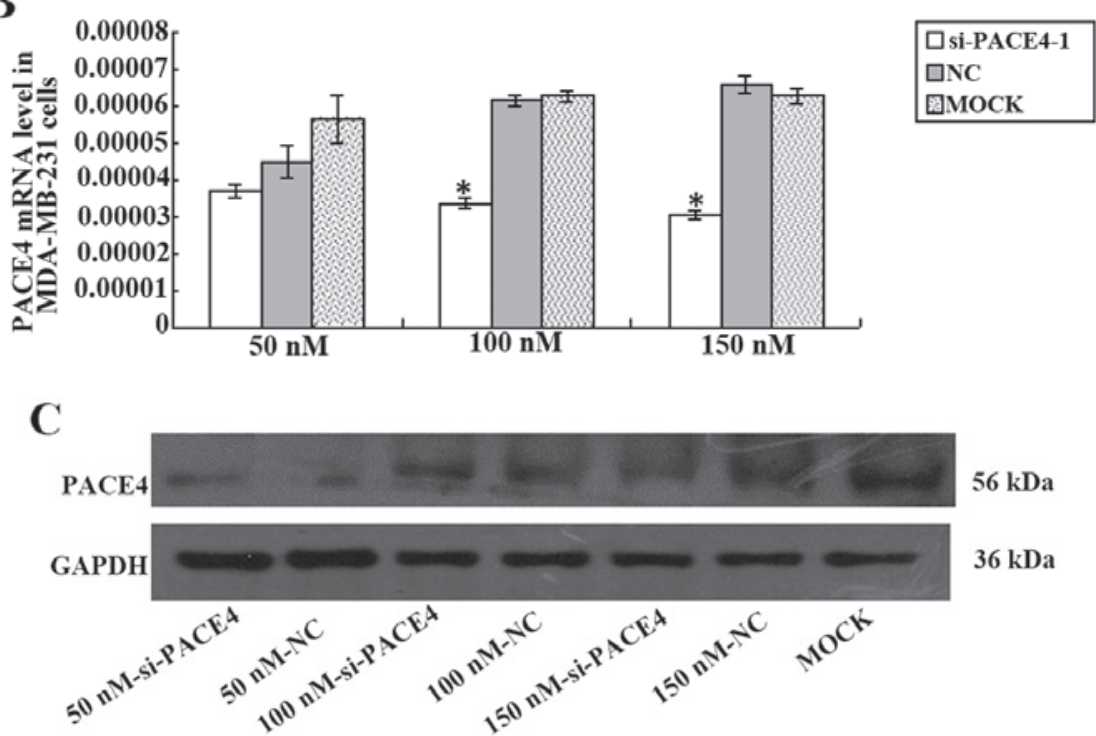

Figure 1. PACE4 expression in MDA-MB-231 cells following transfection with three different pairs of small interfering RNA (siRNA) (A) at different time-points, and (B) at different concentrations of the most effective siRNA, si-PACE4-1. ${ }^{*} \mathrm{P}<0.05,{ }^{* * *} \mathrm{P}<0.01$, and ${ }^{* * * *} \mathrm{P}<0.001$ compared to the NC and MOCK controls. (C) Western blot analysis was used to measure the PACE4 protein expression level. NC, negative control; MOCK, transfection reagent control; GAPDH, glyceraldehyde 3-phosphate dehydrogenase.

(Solabio, China). The number of cells that had invaded through the membrane was quantified in 5 random fields of a fluorescence microscope (ECLIPSE50i, Nikon, Tokyo, Japan) at X100 magnification.

Cell cycle changes detected by flow cytometry. Cells were plated in 6-well culture plates at a density of $1 \times 10^{5}$ cells $/ \mathrm{ml}$ in $2 \mathrm{ml}$ of DMEM, and were transfected with siRNA as described above. The cells were then harvested at $24 \mathrm{~h}$ by addition of trypsin ( $0.25 \%$; Solarbio), incubation for $5 \mathrm{~min}$ and centrifugation at $500 \mathrm{~g}$ for $10 \mathrm{~min}$. The cells were washed twice with PBS, fixed overnight with $70 \%$ cold ethanol, and stained for $30 \mathrm{~min}$ at $37^{\circ} \mathrm{C}$ with $1 \mathrm{ml}$ of propidium iodide solution (50 $\mu \mathrm{g} / \mathrm{ml}$; Beyotime Institute of Biotechnology), containing $50 \mu \mathrm{g} / \mathrm{ml}$ R Nase A (MacGene Biotechnology, Beijing, China). The cell number at different phases of the cell cycle was determined on a FACScan flow cytometer (BD Biosciences, Franklin Lakes, NJ, USA), and the DNA content was analyzed with the ModFitLT V3.0 software (BD Biosciences).

PACE4 expression induced by $17 \beta$-estradiol. MDA-MB-231 and MCF-7 cells were cultured for 3 days in phenol red-free DMEM medium (MacGene Biotechnology) containing 10\% certified charcoal-stripped-FBS (Biological Industries,
Kibbutz Beit-Haemek, Israel), and were next treated with $10^{-10}, 10^{-9}, 10^{-8}, 10^{-7}$ and $10^{-6} \mathrm{~mol} / \mathrm{l}$ of $17 \beta$-estradiol (SigmaAldrich, St. Louis, MO, USA) for 24 or $36 \mathrm{~h}$. Cells cultured in phenol red-free DMEM medium were used as the control. The mRNA level of PACE4 was quantified by RT-qPCR, as described above.

Statistical analysis. Data were expressed as the mean \pm standard deviation (SD). All results were confirmed in at least three independent experiments. Significant differences among three or more datasets were analyzed by a one-way analysis of variance (ANOVA) using the SPSS 17.0 software (SPSS Inc., Chicago, IL, USA). P-values $<0.05$ were considered to indicate statistically significant differences.

\section{Results}

PACE4 is successfully silenced by siRNA. To select the most effective PACE4-specific siRNA, RT-qPCR (data not shown) and western blot analyses were performed to detect the expression of PACE4 at the mRNA and protein level. We used three pairs of siRNA at concentrations $50,100,150 \mathrm{nM}$, and three periods of transfection, 24, 36 and $48 \mathrm{~h}$. The most efficient silencing of PACE4 was achieved with $150 \mathrm{nM}$ (Fig. 1B and C) of siRNA-1, incubated with the cells for 
A
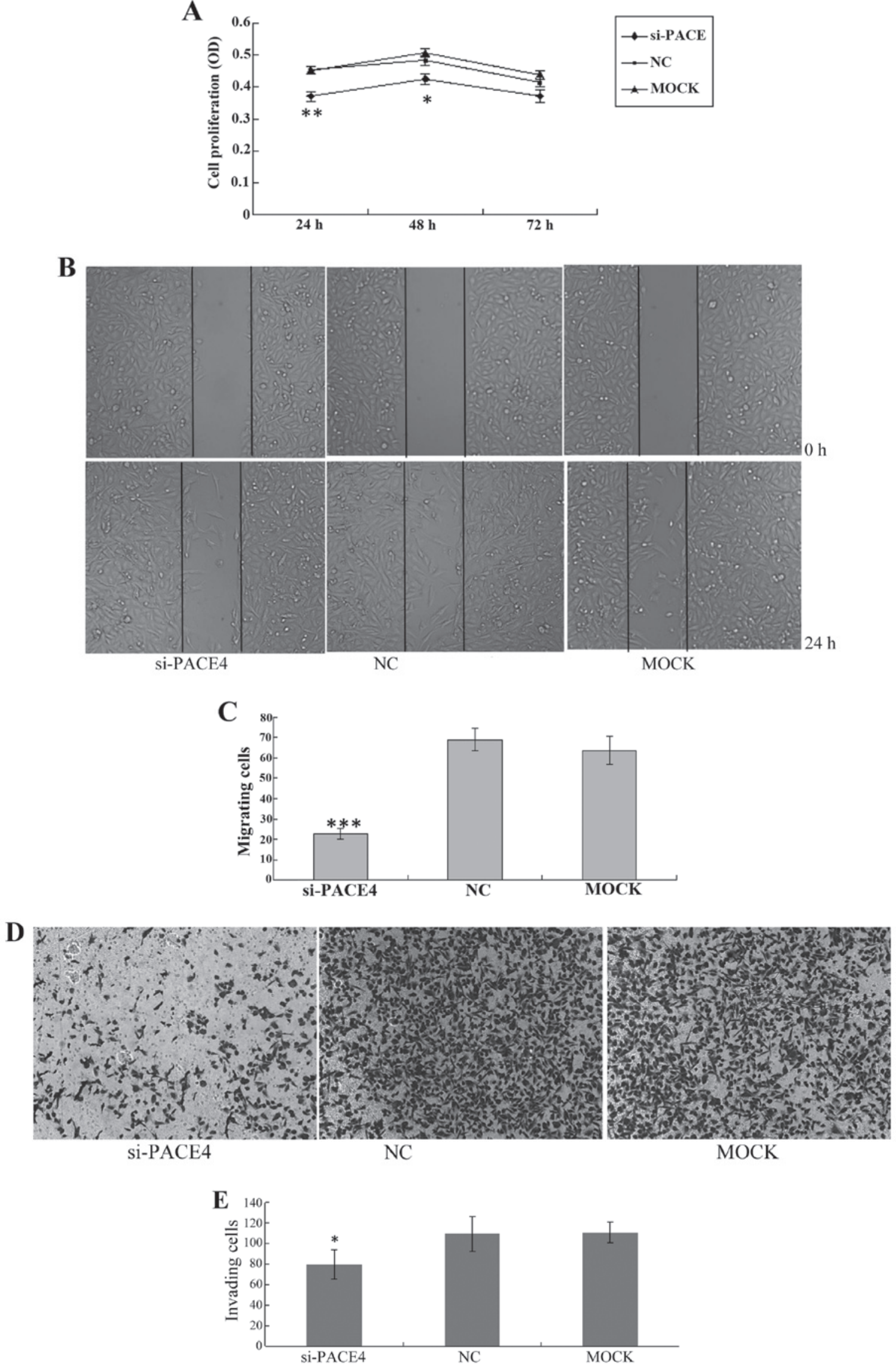

Figure 2. Cell proliferation, migration and invasion in MDA-MB-231 cells transfected with the PACE4 small interfering RNA (siRNA). (A) The MTT assay was used to determine cell proliferation. OD, optical density. (B) Cell migration was detected by a wound healing assay. (D) A Transwell assay was performed to study the cell invasive ability. The average number of cells (C) migrating into the wound and (E) invading through the Transwell membrane was calculated. Three independent experiments were performed. ${ }^{*} \mathrm{P}<0.05,{ }^{* * *} \mathrm{P}<0.01$, and ${ }^{* * *} \mathrm{P}<0.001$, compared to the NC and MOCK controls. NC, negative control; MOCK, transfection reagent control. 


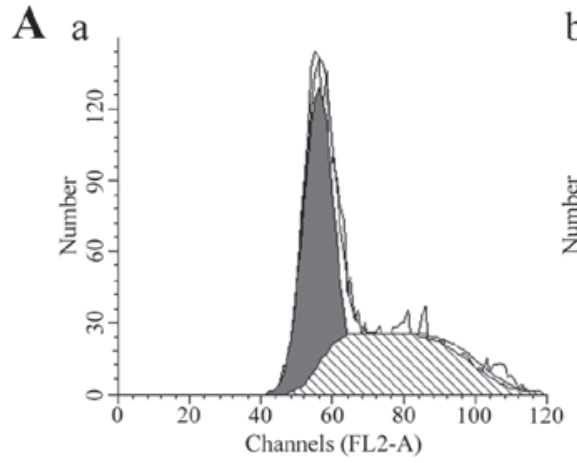

si-PACE4

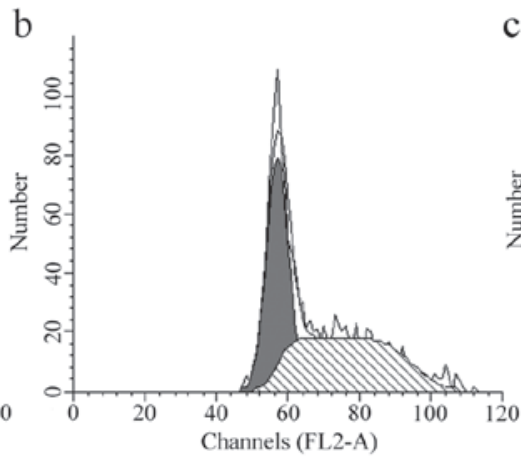

$\mathrm{NC}$

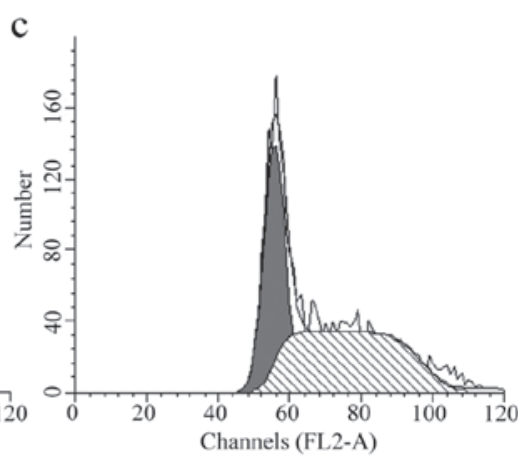

MOCK

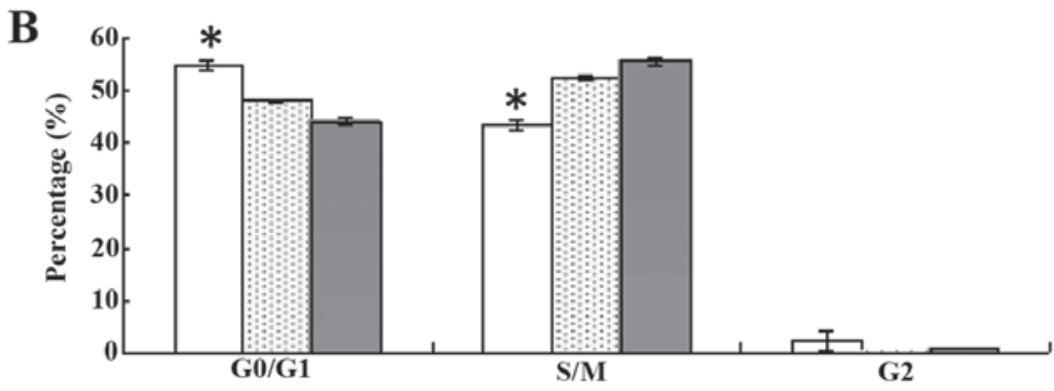

Figure 3. Silencing of PACE4 induces cell-cycle arrest in MDA-MB-231 cells. (A) Representative pictures of flow cytometry and (B) percentage of cells at the different cell-cycle phases. Three independent experiments were performed. Data are expressed as means $\pm \mathrm{SD}$. ${ }^{*} \mathrm{P}<0.05$ compared to the NC and $\mathrm{MOCK}$ controls. NC, negative control; MOCK, transfection reagent control; si-PACE4, small interfering RNA targeting PACE4.

A

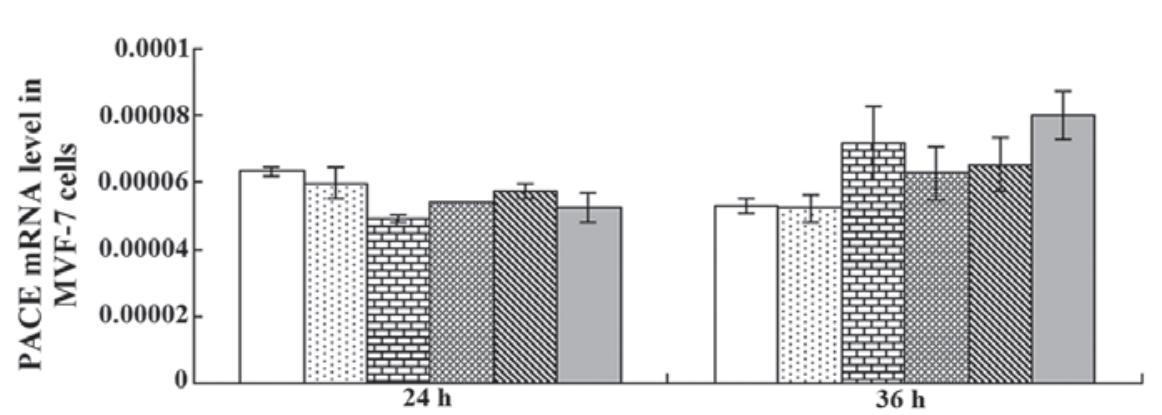

\begin{tabular}{|c|c|}
\hline$\square 10 \mathrm{E}-10$ & 国10E-9 \\
\hline 国10E-8 & 圂10E-7 \\
\hline $\mathbb{N} 10 \mathrm{E}-6$ & $\square$ control \\
\hline
\end{tabular}

B

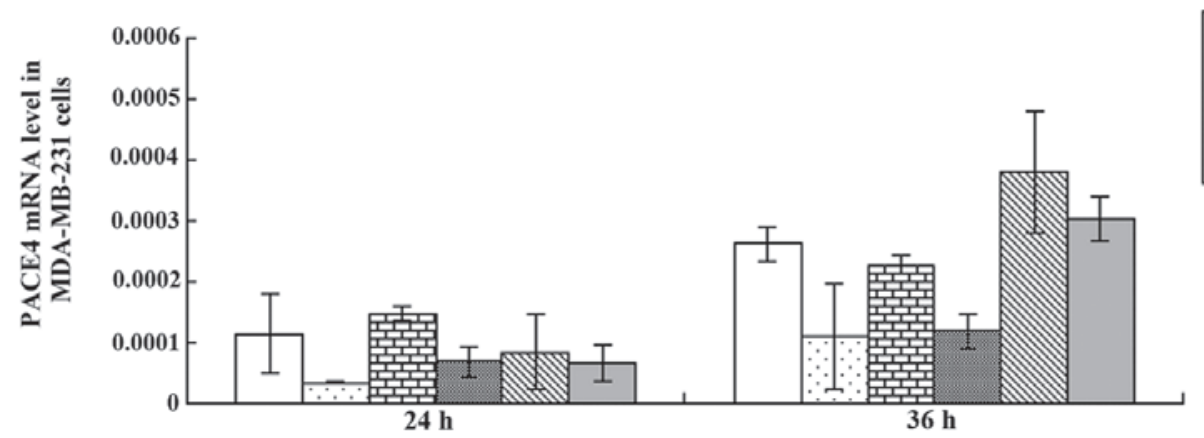

\begin{tabular}{|ll|}
\hline$\square 10 \mathrm{E}-10$ & $\square$ 10E-9 \\
田10E-8 & $\square$ 10E-7 \\
$\mathbb{Q} 10 \mathrm{E}-6$ & $\square$ control \\
\hline
\end{tabular}

Figure 4. mRNA levels of PACE4 in response to 17ß-estradiol treatment. Five estrogen concentrations were tested in (A) MCF-7 cells and (B) MDA-MB-231 cells at 24 and $36 \mathrm{~h}$. Three independent experiments were performed. Data are expressed as means $\pm \mathrm{SD}$. ${ }^{*} \mathrm{P}<0.05$, compared to the control (untreated cells).

$24 \mathrm{~h}$ (Fig. 1A). These conditions were used in the following experiments.

PACE4 silencing decreases the MDA-MB-231 proliferation rate. To analyze cell proliferation following siRNA-mediated silencing of PACE4, the MTT assay was performed. The results showed that the growth rate of the cells transfected with the si-PACE4 is slower than that of the NC and mock control groups (Fig. 2A). This finding indicated that the human breast cancer cell line MDA-MB-231 is sensitive to reduced levels of $P A C E 4$, since silencing of the gene had an inhibitory effect on the growth of these cells. 
A
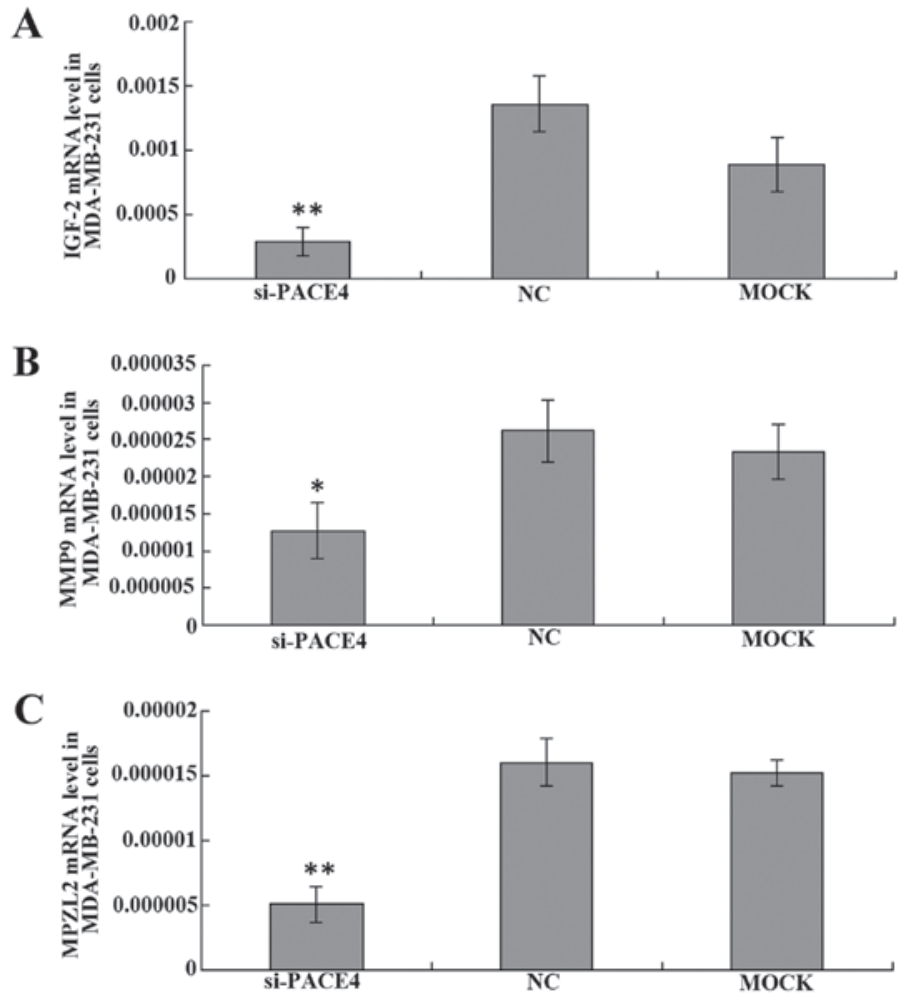

Figure 5. mRNA levels of key genes following PACE4 silencing in MDA-MB-231 cells. The expression of (A) insulin like growth factor 2 (IGF-2), (B) matrix metalloproteinase-9 (MMP9) and (C) myelin protein zero-like 2 (MPZL2) was determined by reverse transcription-quantitative PCR. Three independent experiments were performed. Data are expressed as means $\pm \mathrm{SD}$. ${ }^{*} \mathrm{P}<0.05$, and ${ }^{* *} \mathrm{P}<0.01$, compared to the $\mathrm{NC}$ and MOCK controls. NC, negative control; MOCK, transfection reagent control; si-PACE4, small interfering RNA targeting PACE4.

Silencing of PACE4 reduces $M D A-M B-231$ cell migration and invasion. Cell migration (Fig. 2B) and invasion (Fig. 2D) assays were performed to investigate whether PACE4 affects cell migration and invasion. The results showed that, following PACE4 silencing, cell migration and invasion were both reduced compared to the NC and mock controls. The number of cells that migrated into the wound (Fig. 2C) and invaded through the Matrigel filter (Fig. 2E) were quantified, and were both found to be significantly decreased compared to the controls.

PACE4 may induce G0/G1 phase arrest in breast cancer cells. Flow cytomety was used to investigate the effects of the PACE4 knockdown on the cell cycle. Two types of results are shown in Fig. 3: typical images of flow cytometry showing the cell-cycle phase distribution of the cells (Fig. 3A), and the results of quantification and statistical analyses of cell-cycle phase distribution data at $24 \mathrm{~h}$ (Fig. 3B). These results showed that, following transfection with si-PACE4 for $24 \mathrm{~h}$, the populations of cells at the G0/G1 phase remained increased, which indicates that PACE4 may induce G0/G1 arrest in breast cancer cells.

The expression of PACE4 in response to $17 \beta$-estradiol treatment in MDA-MB-231 and MCF-7 cells. An earlier study showed that the PACE4 gene expression level significantly correlates to the concentration of the cells in estrogen receptors (10). Based on this finding, we investigated whether the expression of PACE4 is affected by $17 \beta$-estradiol using RT-qPCR in the human MDA-MB-231 and MCF-7 cell lines. The results showed that the mRNA level of PACE4 is not significantly different between treatments with different concentrations of $17 \beta$-estradiol (Fig. 4).

The MMP9, IGF-2 and MPZL2 gene levels are decreased following transfection with si-PACE4. To investigate the mechanism underlying the PACE4 silencing-mediated effects on the breast cancer cell line MDA-MB-231, the expression of the $M M P 9, I G F-2$ and $M P Z L 2$ genes was studied following siRNA transfection. qRT-PCR experiments were performed, and the results showed that all three genes show significantly reduced expression following transfection with si-PACE4 (Fig. 5).

\section{Discussion}

In recent years, the relationship between PACE4 and cancer has been extensively studied. Overexpression of PACE4 was demonstrated in skin, lung and prostate cancer $(8,11,12)$. However, other studies reported markedly reduced PACE4 expression levels in human ovarian endometrial cancer cells $(13,14)$. As to breast cancer, PACE4 was found overexpressed, and the study of Lapierre et al (7) demonstrated that overexpression of the prosegment ppPACE4 in the breast cancer cell line MDA-MB-231 significantly enhances cell motility, migration and invasion of collagen in vitro, although the role of PACE4 in these processes was not fully clarified. The abnormal expression of PACE4 has overall become an emerging research focus in numerous cancer studies.

In the present study, we transfected different pairs of siRNA targeting the PACE4 gene (si-PACE4-1 to -4) in the breast cancer cell line MDA-MB-231, and examined the 
degree of PACE4 silencing by RT-qPCR and western blotting. The results showed that the expression of PACE4 is efficiently silenced by si-PACE4. The highest transfection efficiency was achieved at $24 \mathrm{~h}$ of transfection with $150 \mathrm{nM}$ of si-PACE4-1. The MTT assay also indicated that cell growth is prominently decreased at $24 \mathrm{~h}$ of siRNA transfection compared to the control groups. PACE4 has been shown to play a vital role in the activation of numerous growth factors, such as IGF-2. IGF-2 is involved in tumor progression by stimulating cell proliferation, and can be activated by PACE4 at Arg104 (14). We presumed that PACE4 may promote cell proliferation through activation of growth factors such as IGF-2. To confirm this, we studied the expression of the $I G F-2$ gene by RT-qPCR following transfection of MDA-MB-231 cells with si-PACE4, and the result showed that $I G F-2$ expression is significantly decreased in the si-PACE4-transfected cells. We also found that silencing of PACE4 blocks the cell cycle transition from the G0/G1 to the $S$ phase. Taken together, these results indicated that si-PACE4 may inhibit cell proliferation.

MMPs are enzymes involved in a number of pathological processes, and play a vital role in tumor invasion and metastasis, by damaging almost all the proteins of the ECM. MMP9 was identified as an important target of PACE4, degrading the ECM and eventually resulting in enhanced invasiveness (15). To investigate the role of PACE4 in the process(es) enhancing the invasive ability of breast cancer MDA-MB-231 cells, we studied the expression of the MMP9 gene by RT-qPCR. THis analysis showed that, following PACE4 silencing, the MMP9 level is significantly decreased. This result is consistent with the findings of Lapierre et al (7), who reported that overexpression of ppPACE4 can increase the MMP9 activity.

The MPZL2 protein, which contains a putative serine phosphorylation site and is specifically regulated during T cell maturation, is believed to mediate cell adhesion through a homophilic interaction (16). The results of another study by our lab on rheumatoid arthritis (unpublished data) also indicated that MPZL2 may be an important target of PACE4. In this study, RT-qPCR detection of the MPZL2 mRNA level showed that its expression is significantly decreased upon si-PACE4 transfection of MDA-MB-231 cells. All the above suggest that MPZL2 may represent an important protein in cell differentiation.

It is well known that the occurrence and development of breast cancer is closely related to estrogens (17). In this study, we chose MCF-7 cells (estrogen receptor-positive cells) and MDA-MB-231 cells (estrogen receptor-negative cells) to explore the relationship between PACE4 gene expression and estrogen content. However, in contrast to a previous study showing that PACE4 expression significantly correlates to the estrogen receptor content (10), our results did not identify any association between these two parameters.

In conclusion, our study showed that silencing of PACE4 can decrease proliferation, migration and invasion in breast cancer cells. An earlier report proposed that PACE4 inhibition may disrupt the function of numerous proteins critical for tumorigenesis, and that attenuating PACE4 overexpression in cancer may constitute a therapeutic approach (18). Therefore, the PACE4 gene may be a promising inhibition target in the context of breast cancer treatment.

\section{Acknowledgements}

The present study was supported by grants from the National Natural Science Foundation of China (81102275), the Science Promotion Foundation of Shandong (2012GSF12115), the Shandong Scientific Instrument Equipment Promotion Transformation Project (2011SJGZ26), and the Natural Science Foundation of Shandong Province (ZR2011CQ028).

\section{References}

1. Seidah NG and Chrétien M: Proprotein and prohormone convertases: a family of subtilases generating diverse bioactive polypeptides. Brain Res 848: 45-62, 1999.

2. Nagahama M, Taniguchi T, Hashimoto E, et al: Biosynthetic processing and quaternary interactions of proprotein convertase SPC4 (PACE4). FEBS Lett 434: 155-159, 1998.

3. Mains RE, Berard CA, Denault JB, et al: PACE4: a subtilisin-like endoprotease with unique properties. Biochem J 321: 587-593, 1997.

4. Jensen RA, Thompson ME, Jetton TL, et al: BRCA1 is secreted and exhibits properties of a granin. Nat Genet 12: 303-308, 1996.

5. Walerych D, Napoli M, Collavin L and Del Sal G: The rebel angel: mutant p53 as the driving oncogene in breast cancer. Carcinogenesis 33: 2007-2017, 2012.

6. Bassi DE, Zhang J, Cenna J, et al: Proprotein convertase inhibition results in decreased skin cell proliferation, tumorigenesis, and metastasis. Neoplasia 12: 516-526, 2010.

7. Lapierre M, Siegfried G, Scamuffa N, et al: Opposing function of the proprotein convertases furin and PACE4 on breast cancer cells' malignant phenotypes: role of tissue inhibitors of metalloproteinase-1. Cancer Res 67: 9030-9034, 2007.

8. D'Anjou F, Routhier S, Perreault JP, et al: Molecular validation of PACE4 as a target in prostate cancer. Transl Oncol 4: 157-172, 2011.

9. Tsuji A, Sakurai K, Kiyokage E, et al: Secretory proprotein convertases PACE4 and PC6A are heparin-binding proteins which are localized in the extracellular matrix. Potential role of PACE4 in the activation of proproteins in the extracellular matrix. Biochim Biophys Acta 1645: 95-104, 2003.

10. Cheng M, Watson PH, Paterson JA, et al: Pro-protein convertase gene expression in human breast cancer. Int J Cancer 71: 966-971, 1997.

11. Hubbard FC, Goodrow TL, Liu SC, et al: Expression of PACE4 in chemically induced carcinomas is associated with spindle cell tumor conversion and increased invasive ability. Cancer Res 57: 5226-5231, 1997.

12. Schalken JA, Roebroek AJ, Oomen PP, et al: fur gene expression as a discriminating marker for small cell and nonsmall cell lung carcinomas. J Clin Invest 80: 1545-1549, 1987.

13. Fu Y, Campbell EJ, Shepherd TG and Nachtigal MW: Epigenetic regulation of proprotein convertase PACE4 gene expression in human ovarian cancer cells. Mol Cancer Res 1: 569-576, 2003

14. Duguay SJ, Jin Y, Stein J, et al: Post-translational processing of the insulin-like growth factor-2 precursor. Analysis of O-glycosylation and endoproteolysis. J Biol Chem 273: 18443-18451, 1998.

15. Bassi DE, Lopez De Cicco R, Cenna J, et al: PACE4 expression in mouse basal keratinocytes results in basement membrane disruption and acceleration of tumor progression. Cancer Res 65: 7310-7319, 2005.

16. Guttinger M, Sutti F, Panigada M, et al: Epithelial V-like antigen (EVA), a novel member of the immunoglobulin superfamily, expressed in embryonic epithelia with a potential role as homotypic adhesion molecule in thymus histogenesis. J Cell Biol 141: 1061-1071, 1998.

17. Dong S, Zhang Z and Takahara H: Estrogen-enhanced peptidylarginine deiminase type IVgene (PADI4) expression in MCF-7 cells is mediated estrogen receptor-alpha-promoted transfactors activator protein-1, nuclear factor-Y, and Sp1. Mol Endocrinol 21: 1617-1629, 2007.

18. Fu J, Bassi DE, Zhang J, et al: Enhanced UV-induced skin carcinogenesis in transgenic mice overexpressing proprotein convertases. Neoplasia 15: 169-179, 2013. 